\title{
A time-Series Model Based on Quanntum Walk in terms of Quantum Bernoulli Noise
}

\author{
Caiyun Zhang, Caishi Wang, Fangqing Zhang, Xiling Zhang
}

\begin{abstract}
Time-series models play an important role in the research of economics and finance. The QBN-based walk is a new quantum walk model recently introduced in terms of quantum Bernoulli noise. In this paper, we construct a time-series model based on the QBN-based walk and illustrate its basic properties.
\end{abstract}

Index Terms - Time-series; Quantum walk; Quantum Bernoulli noise

MSC(2010):- , 47B38, $60 \mathrm{H} 40$

\section{INTRODUCTION}

As quantum analogs of the classical random walk, quantum walks (also known as quantum random walks) were introduced in 1993 and have been extensively studied since around 2000 (see, e.g [1, 3]). Due to their non-classical properties (for example, ballistic spreading, anti-bell shaped limit density, and localization),quantum walks have found wide application in many fields such as quantum information, quantum computing, and biological systems (see, e.g. [5, 6] and references therein). On the other hand, time-series models such as the ARMA or GARCH play an important role in the research of eco- nomics and finance [2]. Recently, by using quantum walks with finite number degrees of freedom, Konno [4] has introduced a new time-series model and shown its interesting properties.

Quantum Bernoulli noise is the family of annihilation and creation operators acting on Bernoulli functionals, which satisfy the anti-commutation relations $(\mathrm{ACR})$ in equal time, and can provides an approach to the effects of environment on an open quantum system. In 2016, a quantum walk model with infinitely many degrees of freedom, which will be called the QBN-based walk below, was introduced in terms of quantum Bernoulli noise [9]. It has been shown [9] that the QBN-based walk exhibits a rather classical behavior in the limit, which makes it different from the usual quantum walks with finite number degrees of freedom.

In this paper, motivated by the recent work of Konno [4], we would like to construct a time-series model in terms of the QBN-based walk and illustrate its basic properties.Throughout this paper, $\mathrm{Z}$ always denotes thesetof all integers, while $\mathrm{N}$ means the Set of all nonnegative integers. We denote by $\Gamma$ the finite power set of $\mathrm{N}$, namely

Caiyun Zhang, Collage of Mathematics and Statistics, Northwest Normal University, Lanzhou, China, Mobile 86- 18909406395.

Caishi Wang, Collage of Mathematics and Statistics, Northwest Normal University, Lanzhou, China.

Fangqing Zhang, Collage of Mathematics and Statistics, Northwest Normal University, Lanzhou, China.

Xiling Zhang, Collage of Mathematics and Statistics, Northwest Normal University, Lanzhou, China.
$\Gamma=\{\sigma \mid \sigma \subset \mathrm{N}$ and $\# \sigma<\infty\}, \quad(1.1)$

where $\# \sigma$ means the cardinality of $\sigma$. Unless otherwise stated, letters like $\mathrm{j}, \mathrm{k}$ and $\mathrm{n}$ stand for nonnegative integers, namely elements of $\mathrm{N}$.

\section{THE QBS-BASED WALK}

In this section, we briefly recall quantum Bernoulli noise [7], and the quantum walk introduced in [9], namely the QBN-based walk mentioned above.

\subsection{Quantum Bernoulli noise}

Let $\Omega=\{-1,1\}^{N}$ be the set of all mapping $\omega: N \mapsto\{-1,1\}$, and $\left(\zeta_{n}\right)_{n>0}$ the sequences of canonical projections on $\Omega$ given by

$$
\zeta_{n}(\omega)=\omega(n), \quad \omega \in \Omega
$$

Let $\mathfrak{I}$ be the $\sigma$-field on $\Omega$ generated by the sequences $\left(\zeta_{n}\right)_{n \geq 0}$, and $\left(p_{n}\right)_{n \geq 0}$ a given sequences of positives numbers with the property that $0<p_{n}<1$ for all $n \geq 0$.then there exists a unique probability measure $\mathrm{P}$ on $\mathfrak{I}$ such that

$$
\begin{aligned}
& P \circ\left(\zeta_{n 1}, \zeta_{n 2}, \ldots \zeta_{n k}\right)^{-1}\left\{\left(\varepsilon_{1}, \varepsilon_{2}, \ldots, \varepsilon_{\mathrm{k}}\right)\right\} \\
& =\prod_{\mathrm{j}=1}^{\mathrm{k}} \mathrm{p}_{\mathrm{j}}^{\frac{1+\varepsilon_{\mathrm{j}}}{2}}\left(1-p_{j}\right)^{\frac{1-\varepsilon_{\mathrm{j}}}{2}}
\end{aligned}
$$

for $n_{j} \in N, \varepsilon_{j} \in\{-1,1\}(1 \leq j \leq k)$ with $n_{i} \neq n_{j}$ when $i \neq j$ and $k \in N$ with $k \geq 1$. Thus one has a probability

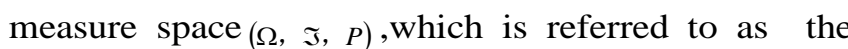
Bernoulli space and random variables on it are known as Bernoulli functionals.

Let $Z_{n}=\left(Z_{n}\right)_{n \geq 0}$ be the sequences of Bernoulli functionals defined by

$$
Z_{n}=\frac{\zeta_{n}+q_{n}+p_{n}}{2 \sqrt{q_{n} p_{n}}}, n \geq 0
$$

where $q_{n}=1-p_{n} \quad$.Clearly $Z_{n}=\left(Z_{n}\right)_{n \geq 0} \quad$ is $\quad$ an independent sequence of random variables on the probability measure $\operatorname{space}(\Omega, \mathfrak{I}, P)$.

Let $H$ be the space of square integrable complex-valued Bernoulli functionals, namely

$$
H=L^{2}(\Omega, \mathfrak{R}, P)
$$

We denote by $\langle\cdot, \cdot\rangle$ the usual inner product of the space $H$, and by $\|\cdot\|$ the corresponding norm. It is known that $\mathrm{Z}$ has the chaotic representation property, which means that the family $\left\{Z_{\sigma} \mid \sigma \in \Gamma\right\}$ forms an orthonormal basis of $\mathrm{H}$, where $Z_{\varnothing}=1$ and 


$$
Z_{\sigma}=\prod_{j \in \sigma} Z_{j}, \quad \sigma \in \Gamma, \sigma \neq \varnothing .
$$

In the following, we call $\left\{Z_{\sigma} \mid \sigma \in \Gamma\right\}$ the canonical ONB of $\mathrm{H}$. Clearly $\mathrm{H}$ is infinitely dimensional since $\left\{Z_{\sigma} \mid \sigma \in \Gamma\right\}$ is countably infinite.

Lemma 2.1. [7] For each $k \in N$, there exists a bounded operator $\partial_{k}$ on $\mathrm{H}$ such that

$$
\partial_{k} Z_{\sigma}=1_{\sigma}(k) Z_{\sigma \backslash k}, \quad \sigma \in \Gamma
$$

where $\sigma \backslash \mathrm{k}=\sigma \backslash\{\mathrm{k}\}$ and $\mathbf{1}_{\sigma}(\mathrm{k})$ the indicator of $\sigma$ as a subset of $\mathrm{N}$.

Lemma 2.2.[7] Let $k \in N$.Then $\partial_{k}^{*}$, the adjoint of operator $\partial_{k}$, has following property:

$$
\partial_{k}^{*} Z_{\sigma}=\left[1-1_{\sigma}(k)\right] Z_{\sigma \cup k}, \quad \sigma \in \Gamma
$$

where $\sigma \cup \mathrm{k}=\sigma \cup\{\mathrm{k}\}$.

Remark. The family $\left\{\partial_{k}, \partial_{k}^{*}\right\}_{k \geq 0}$ is called quantum Bernoulli noise, while $\partial_{k}$ and $\partial_{k}^{*}$ are known as the annihilation and creation operators, respectively.

Lemma 2.3. [9] For nonnegative integer $n \geq 0$, define operators $\mathrm{L}_{\mathrm{n}}$ and $\mathrm{R}_{\mathrm{n}}$ on $\mathrm{H}$ as

$$
L_{\mathrm{n}}=\frac{1}{2}\left(\partial_{n}^{*}+\partial_{n}-I\right), \quad R_{\mathrm{n}}=\frac{1}{2}\left(\partial_{n}^{*}+\partial_{n}+I\right),
$$

where $\mathrm{I}$ is the identity operator on $\mathrm{H}$. Then both $\mathrm{L}_{n}$ and $\mathrm{R}_{n}$ are self-adjoint, and moreover they admit the following properties

$$
L_{n}^{2}=-L_{n}, L_{n} R_{n}=R_{n} L_{n}, R_{n}^{2}=R_{n} .
$$

\subsection{The QBN-based walk}

Let $l^{2}(Z, H)$ be the space of square summable functions defined on $\mathrm{Z}$ and valued in $\mathrm{H}$, namely

$$
l^{2}(Z, H)=\left\{\Phi: Z \rightarrow H \mid \sum_{x=-\infty}^{\infty}\|\Phi(x)\|^{2}<\infty\right\} .
$$

Then $l^{2}(Z, H)$ remains a complex Hilbert space, whose inner product $\langle\cdot \cdot\rangle_{l^{2}(Z, H)}$ is given by

$$
\begin{aligned}
& \langle\Phi, \Psi\rangle_{l^{2}(Z, H)}=\sum_{x=-\infty}^{\infty}\langle\Phi(x), \Psi(x)\rangle, \\
& \Phi, \Psi \in l^{2}(Z, H),
\end{aligned}
$$

where $\langle\cdot\rangle$,$\rangle denotes the inner product of \mathrm{H}$ as indicated in Section 2.1. By convention, we denote by $\|\cdot\|_{l^{2}(Z, H)}$ the norm induced by $\langle\cdot, \cdot\rangle_{l^{2}(Z, H)}$. As usual, a vector $\Phi \in l^{2}(Z, H)$ is called normalized if . $\|\Phi\|_{l^{2}(Z, H)}=1$

Deftnition 2.1. [9] The QBN-based walk is a quantum walk on $\mathrm{Z}$ that satisfies the following requirements

- The state space of the walk is $l^{2}(Z, H)$ and its states are represented by normalized vectors in $l^{2}(Z, H)$.

- The time evolution of the walk is governed by equation

$$
\Phi_{n+1}(x)=R_{n} \Phi_{n}(x-1)+L_{n} \Phi_{n}(x+1), \quad x \in Z, n \geq 0
$$

where $\Phi_{n} \in l^{2}(Z, H)$ denotes the state of the walk at time $n \geq$ 0 .

Let $\left(\Phi_{n}\right)_{n \geq 0}$ be the state sequence of the QBN-based walk. That the function $x \mapsto\left\|\Phi_{n}(x)\right\|^{2}$ makes a probability distribution on $\mathrm{Z}$, which is called the probability distribution of the walk at time $n \geq 0$. In particular, $\left\|\Phi_{n}(x)\right\|^{2}$ is the probability that the quantum walker is found at position $x \in Z$ at time $n \geq 0$.

As usual, the QBN-based walk is assumed to start at position $x=0$, which implies that its initial state $\Phi_{0}(x)$ is a localized one, namely $\Phi_{0}$ is such that $\Phi_{0}(x)=0$ for $x \in Z$ with $x \neq 0$. Thus $\Phi_{0}(x)$ plays an important role in investigating the asymptotic behavior of the walk.

It is well known that $l^{2}(Z, H) \cong l^{2}(Z) \otimes H$. This just means that $l^{2}(Z)$ describes the position of the QBN-based walk, while $\mathrm{H}$ describes its internal degrees of freedom. By convention, $\mathrm{H}$ is called the coin space of the QBN-based walk.

Remark. The QBN-based walk has infinitely many internal degrees of freedom, since its coin space $\mathrm{H}$ is infinitely dimensional.

Lemma 2.4. [9] Let the initial state $\Phi_{0}$ of the $Q B N$-based walk be such that

$\Phi_{0}(x)=\left\{\begin{array}{r}\alpha Z_{\varnothing}+\beta Z_{0}, x=0 \\ 0, x \neq 0, x \in Z\end{array}\right.$

where $\alpha, \beta \in C$ with $|\alpha|^{2}+|\beta|^{2}=1$. Then, for all $n \geq 1$, one has

$$
\begin{aligned}
& \left\|\Phi_{n}(x)\right\|^{2} \\
& =\left\{\begin{array}{l}
\left.\frac{1}{2^{n}}\left[\begin{array}{l}
n-1 \\
j
\end{array}\right)|\alpha-\beta|^{2}+\left(\begin{array}{l}
n-1 \\
j-1
\end{array}\right)|\alpha+\beta|^{2}\right], x=2 j-1,0 \leq j \leq n \\
0, x \neq 0, x \in Z
\end{array}\right. \\
& \text { where }\left(\begin{array}{l}
n-1 \\
-1
\end{array}\right)=\left(\begin{array}{l}
n-1 \\
n
\end{array}\right)=0, \text { and }\left(\begin{array}{l}
0 \\
0
\end{array}\right)=1 .
\end{aligned}
$$

\section{TIME-SERIES MODEL IN TERMS OF THE QBN-BASED WALK}

In this section, we construct a time-series model in terms of the QBN-based walk, and give several examples to illustrate its properties

Assume that $D_{n}=\left\{x_{1}, x_{2}, \cdots x_{n}\right\}$ is a set of real-valued time-series data until time $n$ If $D_{n}$ is given, we want to estimate a next time $x_{n+1}$ using a framework of the QBNbased walk with localized initial state $\Phi_{0}(0)=\theta Z_{\varnothing}+2 \sqrt{1-\theta^{2}} Z_{0}$, where $\theta \in \Theta=[0,1]$ is the parameter. To do so, we introduce the following time-dependent evaluation function

$$
V_{n}(\theta)=\sum_{k=0}^{n} \sum_{x=-k}^{k}\left(x-x_{k}\right)^{2}\left\|\Phi_{k}(x)\right\|^{2} \text { (3.1) }
$$

where $\sum_{x=-k}^{x=k}$ means to sum for all integers from $-k$ to $k$. 
Note that, by Lemma $2.4, \Phi_{k}(x) \|^{2}$ has the following representatio

$$
\begin{aligned}
& \left\|\Phi_{n}(x)\right\|^{2} \\
& = \begin{cases}\frac{1}{2^{k}}\left[\left(\begin{array}{l}
k-1 \\
j
\end{array}\right)\left(1-2 \theta \sqrt{1-\theta^{2}}\right)+\left(\begin{array}{c}
k-1 \\
j-1
\end{array}\right)\left(1+2 \theta \sqrt{1-\theta^{2}}\right)\right], x=2 j-k .0 \leq j \leq k ; \\
0, & \text { otherwise }\end{cases} \\
& \text { where }\left(\begin{array}{c}
k-1 \\
-1
\end{array}\right)=\left(\begin{array}{l}
k-1 \\
k
\end{array}\right)=0,\left(\begin{array}{l}
0 \\
0
\end{array}\right)=1 \text { and } 0 \leq k \leq n .
\end{aligned}
$$

Without loss of generality, we may assume that $x_{0}=0$.Then we can take the following algorithm to estimate $x_{n+1}$ from the given data $D_{n}=\left\{x_{0}, x_{1}, \cdots, x_{n}\right\}$.

- Step 1. Find a $\theta_{n}^{*} \in \Theta$ such that the function $V_{n}(\theta)$ attains its minimum at $\theta=\theta_{n}^{*}$.

- Step 2. For the $\theta_{n}^{*}$ given in Step 1, compute $E\left(X_{n+1}\right)$, the expectation of theposition $X_{t}$ of the quantum walker at time $t=n+1$. If $\theta_{n}^{*}$ is uniquely determined, then $E\left(X_{n+1}\right)$ is taken as the estimated value of $x_{n+1}$, namely $x_{n+1}^{*}=E\left(X_{n+1}\right)$. If there are some options of $\theta_{n}^{*}$, then just put $x_{n+1}^{*}$ as the average of $E\left(X_{n+1}\right)$ over these choices. If $V_{V_{n}}(\theta)$ is a constant for any $\theta \in \Theta$, then simply put $x_{n+1}^{*}=x_{n}$.

- Step 3. By repeating above procedures, Steps 1 and 2, one obtains a sequence of estimated values $\left\{x_{1}^{*}, x_{2}^{*}, \cdots x_{n}^{*}\right\}$.

Example 3.1. The case of $n=0$. In this case, we want to estimate $x_{1}$ from the given data $D=\left\{x_{0}\right\}$ with $x_{0}=0 . A$ simple calculation gives

$$
V_{0}(\theta)=\left(0-x_{0}\right)^{2}\left\|\Phi_{0}(0)\right\|^{2}=0, \quad \theta \in \Phi
$$

Thus, by the algorithm $x_{1}^{*}=x_{0}=0$, namely the estimated value of $x_{1}$ is 0 .

Example 3.2. The case of $n=1$. In this case, we want to estimate $x_{2}$ from the given data $D=\left\{x_{0}, x_{1}\right\}$ with $x_{0}=0$. By a lengthy calculation, we can get the evaluation function as follows.

$$
\begin{aligned}
V_{1}(\theta) & =\left(-1-x_{1}\right)^{2}\left\|\Phi_{1}(-1)\right\|^{2}+\left(0-x_{1}\right)^{2}\left\|\Phi_{1}(0)\right\|^{2}+\left(1-x_{1}\right)^{2}\left\|\Phi_{1}(1)\right\|^{2} \\
& =\frac{1}{2}\left(1+x_{1}\right)^{2}\left(1-2 \theta \sqrt{1-\theta^{2}}\right)+\frac{1}{2}\left(1-x_{1}\right)^{2}\left(1+2 \theta \sqrt{1-\theta^{2}}\right) \\
& =1+x_{1}^{2}-4 x_{1} \theta \sqrt{1-\theta^{2}}
\end{aligned}
$$

\begin{tabular}{lll}
\hline$x_{0}$ & $x_{1}$ & $x_{2}^{*}$ \\
\hline$x_{0}=0$ & $x_{1}>0$ & $x_{2}^{*}=1$ \\
$x_{0}=0$ & $x_{1}=0$ & $x_{2}^{*}=x_{1}=0$ \\
$x_{0}=0$ & $x_{1}<0$ & $x_{2}^{*}=0$
\end{tabular}

Table 1:the values of $x_{0}, x_{1}$ and $x_{2}^{*}$

where $\theta \in \Theta=[0,1]$. We consider three cases: (1) $x_{1}>$ 0 ; (2) $x_{1}=0$; (3) $x_{1}<0$

Case(1): $x_{1}>0$ In this case, $V_{1}(\theta) \leq 1+x_{1}^{2}=V_{1}(0)=V_{1}(1)$ which implies that $V_{1}(\theta)$ attains its minimum at a unique $\theta_{1}^{*}=\frac{\sqrt{2}}{2} \in \Theta$.Thus, by the algorithm we have

$$
\begin{aligned}
x_{2}^{*}=E\left(X_{2}\right)= & (-2) \times\left\|\Phi_{2}(-2)\right\|^{2}+0 \times\left\|\Phi_{2}(0)\right\|^{2}+2 \times\left\|\Phi_{2}(2)\right\|^{2} \\
= & (-2) \times \frac{1}{4}\left(1-2 \theta_{1}^{*} \sqrt{1-\left(\theta_{1}^{*}\right)^{2}}\right)+ \\
& 2 \times \frac{1}{4}\left(1+2 \theta_{1}^{*} \sqrt{1-\left(\theta_{1}^{*}\right)^{2}}\right) \\
= & 1
\end{aligned}
$$

Case (2): $x_{1}=0$. Clearly $V_{1}(\theta)=1$ for all $\theta \in \Theta$. Thus $x_{2}^{*}=x_{1}=0$.

Case (3): $x_{1}<0$. It is easy to see that $V_{1}(\theta) \geq 1+x_{1}^{2}=V_{1}(0)=V_{1}(1)$, which means that $V_{1}(\theta)$ attains its minimum at $\theta_{1}^{*}=0$ or $\theta_{1}^{*}=1$ For $\theta_{1}^{*}=0$, we find

$$
\begin{aligned}
E\left(X_{2}\right) & =(-2) \times\left\|\Phi_{2}(-2)\right\|^{2}+0 \times\left\|\Phi_{2}(0)\right\|^{2}+2 \times\left\|\Phi_{2}(2)\right\|^{2} \\
& =(-2) \times \frac{1}{4}\left(1-2 \theta_{1}^{*} \sqrt{1-\left(\theta_{1}^{*}\right)^{2}}\right)+2 \times \frac{1}{4}\left(1+2 \theta_{1}^{*} \sqrt{1-\left(\theta_{1}^{*}\right)^{2}}\right) \\
& =2 \theta_{1}^{*} \sqrt{1-\left(\theta_{1}^{*}\right)^{2}} \\
& =0
\end{aligned}
$$

similarly, for $\theta_{1}^{*}=1$, we still have $E\left(X_{2}\right)=0$. Thus $x_{2}^{*}=\frac{1}{2} \times 0+\frac{1}{2} \times 0=0$.Therefore, we get the estimated value $x_{2}^{*}$ of $x_{2}$ for all cases. To summarize, we have Table 1.

Example 3.3. The case of $n=2$. In this case, we want to estimate $x_{3}$ from the given data $D=\left\{x_{0}, x_{1}, x_{2}\right\}$ with $x_{0}=0$. Again by a lengthy calculation we can come to

$V_{2}(\theta)$

$=V_{1}(\theta)+\left(-2-x_{2}\right)^{2} \times\left\|\Phi_{2}(-2)\right\|^{2}+\left(0-x_{2}\right)^{2} \times\left\|\Phi_{2}(0)\right\|^{2}+\left(2-x_{2}\right)^{2} \times\left\|\Phi_{2}(2)\right\|^{2}$ $=V_{1}(\theta)+\frac{1}{4}\left(2+x_{2}\right)^{2}\left(1-2 \theta_{1} \sqrt{1-\theta_{1}^{2}}\right)+\frac{1}{2} x_{2}^{2}+\frac{1}{4}\left(2-x_{2}\right)^{2}\left(1+2 \theta_{1} \sqrt{1-\theta_{1}^{2}}\right)$

$=3+x_{1}^{2}+x_{2}^{2}-4\left(x_{1}+x_{2}\right) \theta_{1}^{*} \sqrt{1-\theta_{1}^{* 2}}$

where $\theta \in \Theta=[0,1]$. We consider three cases: (1) $x_{1}+x_{2}>$ 0; (2) $x_{1}+x_{2}=0$; (3) $x_{1}+x_{2}<0$.

Case (1): $\quad x_{1}+x_{2}>0 \quad$ In this case, $V_{2}(\theta) \leq 3+x_{1}^{2}+x_{2}^{2}=V_{2}(0)=V_{2}(1)$ which implies that $V_{2}(\theta)$ attains its minimum at a unique $\theta_{1}^{*}=\frac{\sqrt{2}}{2} \in \Theta$.Thus, by the algorithm we have

\begin{tabular}{ccccc}
\hline$x_{0}$ & $x_{1}$ & $x_{2}$ & $x_{1}+x_{2}$ & $x_{3}^{*}$ \\
\hline$x_{0}=0$ & $x_{1}$ & $x_{2}$ & $x_{1}+x_{2}>0$ & $x_{3}^{*}=1$ \\
$x_{0}=0$ & $x_{1}$ & $x_{2}$ & $x_{1}+x_{2}=0$ & $x_{3}^{*}=x_{2}$ \\
$x_{0}=0$ & $x_{1}$ & $x_{2}$ & $x_{1}+x_{2}<0$ & $x_{3}^{*}=0$
\end{tabular}

Table 2:the values of $x_{0}, x_{1}, x_{2}$ and $x_{3}^{*}$ 


$$
\begin{aligned}
x_{3}^{*} & =E\left(X_{3}\right) \\
& =(-3) \times\left\|\Phi_{3}(-3)\right\|^{2}+(-1) \times\left\|\Phi_{2}(-1)\right\|^{2}+1 \times\left\|\Phi_{2}(1)\right\|^{2}+3 \times\left\|\Phi_{3}(3)\right\|^{2} \\
& =(-3) \times 0+(-1) \times \frac{1}{4}+1 \times \frac{1}{2}+3 \times \frac{1}{4} \\
& =1
\end{aligned}
$$

Case (2): $x_{1}+x_{2}=0$. In this case, $V_{2}(\theta)=3+x_{1}^{2}+x_{2}^{2}$ for all $\theta \in \Theta=[0,1]$. Thus $x_{3}^{*}=x_{2}$

Case(3): $\quad x_{1}+x_{2}<0$. In this case, $V_{2}(\theta) \geq 3+x_{1}^{2}+x_{2}^{2}=V_{2}(0)=V_{2}(1)$, which means that $V_{2}(\theta)$ attains its minimum at $\theta_{2}^{*}=0$ or $\theta_{2}^{*}=1$. For $\theta_{2}^{*}=0$, we have

$$
\begin{aligned}
& E\left(X_{3}\right) \\
& =(-3) \times\left\|\Phi_{3}(-3)\right\|^{2}+(-1) \times\left\|\Phi_{3}(-1)\right\|^{2}+1 \times\left\|\Phi_{3}(1)\right\|^{2}+3 \times\left\|\Phi_{3}(3)\right\| \\
& =(-3) \times \frac{1}{8}+(-1) \times \frac{3}{8}+1 \times \frac{3}{8}+3 \times \frac{1}{8} \\
& =0
\end{aligned}
$$

Similarly, for $\theta_{2}^{*}=1$, we still have $E\left(X_{3}\right)=0$. Thus $x_{3}^{*}=\frac{1}{2} \times 0+\frac{1}{2} \times 0=0$

Therefore, we have the estimated value $x_{3}^{*}$ of $x_{3}$ for all cases. For summary, we give Table 2.

\section{ACKNOWLEDGEMENT}

This work is supported by National Natural Science Foundation of China (Grant No. 11461061 and Grant No. 11861057).

\section{REFERENCES}

[1] Y. Aharonov, L. Davidovich and N. Zagury, Quantum random walks. Phys. Rev. A 48 (1993), 1687-1690.

[2] G.E.P. Box and G.M. Jenkins, Time Series Analysis:Forecasting and Control, Holden-Day, San Francisco (1970).N.

[3] Konno, Quantum walks, In: Franz, U., Schürmann, M. (eds.) Quantum Potential Theory, Lecture Notes in Mathematics, vol. 1954, pp. 309-452. Springer, Heidelberg (2008)

[4] N. Konno, A new time-series model based on quantum walk Quantum Stud.: Math.Found. (2018).https://doi.org/10.1007/s40509-018-0162-1

[5] R. Portugal, Quantum Walks and Search Algorithms, Springer, Berlin (2013)

[6] S.E. Venegas-Andraca, Quantum walks: a comprehensive review. Quantum Inf. Pro- cess. 11 (2012), 1015-1106.

[7] C.S. Wang, H.F. Chai and Y.C. Lu, Discrete-time quantum Bernoulli noises, J. Math. Phys. 51 (2010), no. 5, 053528.

[8] C.S. Wang, Y.C. Lu, and H.F. Chai, An alternative approach to Privault's discrete- time chaotic calculus, J. Math. Anal. Appl. 373 (2011), 643-654.

[9] C.S. Wang and X.J. Ye, Quantum walk in terms of quantum Bernoulli noises, Quan- tum Inf. Process. 15 (2016), no. 5, 1897-1908.
Caiyun Zhang, Collage of Mathematics and Statistics, Northwest Normal University, Lanzhou, China, Mobile 86- 18909406395.

Caishi Wang, Collage of Mathematics and Statistics, Northwest Normal University, Lanzhou, China.

Fangqing Zhang, Collage of Mathematics and Statistics, Northwest Normal University, Lanzhou, China.

Xiling Zhang, Collage of Mathematics and Statistics, Northwest Normal University, Lanzhou, China. 